\title{
Technologies for the Realization of the Square Kilometre Array
}

\author{
Jan Geralt Bij de Vaate ${ }^{1}$ \\ ASTRON \\ P.O. Box 2, 7990AA, Dwingeloo, The Netherlands \\ E-mail: vaate@astron.nl
}

\begin{abstract}
A paradigm shift is about to happen in next generation radio telescopes. Where most current antenna systems are based on large reflectors with a single or a small number of feeds, new telescopes will require phased-array solutions or phased-array feed solutions. This requirement is driven by the key science projects described in [1], [2] for frequencies below 10GHz. Besides the requirement for a significantly improved sensitivity, larger patches of the sky need to be observed instantaneously. Enhancing the sensitivity with two orders of magnitude over existing telescopes can be achieved with the realization of a Square Kilometre Array (SKA) collecting area [3], [4]. Enhancing the Field of View, 20 up to 200 square degrees at $1 \mathrm{GHz}$, can only be achieved by processing more beams. This can be realized with a Phased Array Feed (PAF) in the focus of a reflector e.g. forming 25 beams. Or with a full phased array or Aperture Array (AA), where the number of beams only depends on the available processing power.
\end{abstract}

2nd MCCT-SKADS Training School Radio Astronomy: fundamentals and the new instruments Sigüenza (Spain) August $26^{\text {th }}-$ September $4^{\text {th }} 2008$

\footnotetext{
$1 \quad$ Speaker
} 


\section{Introduction}

The specifications for the new generation radio telescopes will be derived from key science projects. To serve all science cases with a single telescope will not be possible or at least will generate a very expensive instrument, therefore choices will need to be made. In [6] (Memo 100), a first short list SKA specification is given, briefly summarized in Table 1.

Table 1 Basic SKA requirements

\begin{tabular}{|l|r|}
\hline Frequency range & $70 \mathrm{MHz}-10 \mathrm{GHz}$ \\
\hline Sensitivity & $12.000 \mathrm{~m}^{2} / \mathrm{K}$ \\
\hline Survey speed & $210^{10} \mathrm{~m}^{4} / \mathrm{K}^{2} \mathrm{deg}^{2}$ \\
\hline
\end{tabular}

The survey speed requirement drives the need for large Fields of View.

This paper will briefly touch upon the capabilities of the new Wide Field of View (WFoV) technological developments.

\section{System Technology Demonstrators}

Research and development for the SKA is performed at over 50 institutes world wide. A large part of this R\&D is focused on the design, realization and verification of demonstrators or SKA pathfinders. The following sections describe a number of these with a focus on Wide Field of View technology. Note that this is only a subset and not a complete coverage of the R\&D projects.

\subsection{Low frequency aperture array: LOFAR}

For the low frequency range of the SKA, $70-400 \mathrm{MHz}$, the use of a reflector type antenna is not a realistic option: only a very large dish can receive these low frequencies efficiently. A pathfinder for this frequency range is the Low Frequency Array (LOFAR) [5], realised in the north of the Netherlands by ASTRON, with international stations in Germany, Sweden, France and the UK. LOFAR consists of two arrays, one for the $30-80 \mathrm{MHz}$ and one for the 120 $240 \mathrm{MHz}$. Figure 1 gives an air view of the first complete station. Not very good visible are the low band antennas which consist of droopy dipoles made of posts and wires. The high band antennas are the flat black boxes in the photograph, antennas in EPS covered with a black environmental protection. The beam of the aperture array LOFAR system electronically formed and processing/correlation is done in a super computer.

Table 2 LOFAR specifications

\begin{tabular}{|l|r|l|}
\hline High band frequency range & $120-240 \mathrm{MHz}$ & \\
\hline Low band frequency range & $30-80 \mathrm{MHz}$ & \\
\hline Collecting area, high band & $62400 \mathrm{~m}^{2}$ & At 120MHz, reduces with $\lambda^{2}$ \\
\hline Collecting area, low band & $60000 \mathrm{~m}^{2}$ & At 30MHz, reduces with $\lambda^{2}$ \\
\hline System temperature, $\mathrm{T}_{\text {sys }}$ & $\mathrm{K}$ & Sky noise dominated \\
\hline Total no. of stations & $36+8$ & LOFAR + international stations \\
\hline Station size & $81 / 31 \mathrm{~m}$ & High band / low band \\
\hline No. of stations in core & 18 & \\
\hline
\end{tabular}




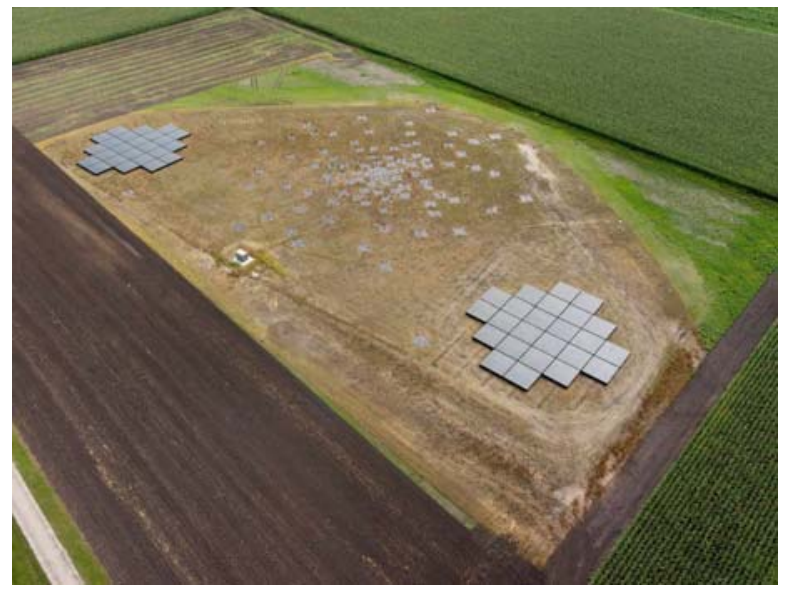

Figure 1 Air photograph of a LOFAR station

\subsection{Mid frequency aperture array: EMBRACE}

A group of 29 European institutes conduct research within the FP-6 programme SKA Design Study (SKADS) [6]. Many aspects of phased array Wide Field of View (WFoV) technologies are being investigated and two AA demonstrators will be built, one is a fully digital array of modest size and the second is a $150 \mathrm{~m}^{2}$ array, the Electronic Multi Beam Radio Astronomy Concept (EMBRACE) [7].

Table 3 EMBRACE specification

\begin{tabular}{|l|l|}
\hline Frequency range & $500-1500 \mathrm{MHz}$ \\
\hline Physical collecting area & $\sim 150 \mathrm{~m} 2$, Westerbork, Netherlands \\
& $\sim 80 \mathrm{~m} 2$ Nançay, France \\
\hline Aperture efficiency & $>80 \%$ \\
\hline Electronic scan range & $+/-45 \mathrm{deg}$ \\
\hline $\mathrm{T}_{\text {sys }}$ & $<100 \mathrm{~K}$ at $1 \mathrm{GHz}$ \\
\hline Instantaneous bandwidth & $200 \mathrm{MHz}$ \\
\hline Number of independent FoV & 2 \\
\hline
\end{tabular}

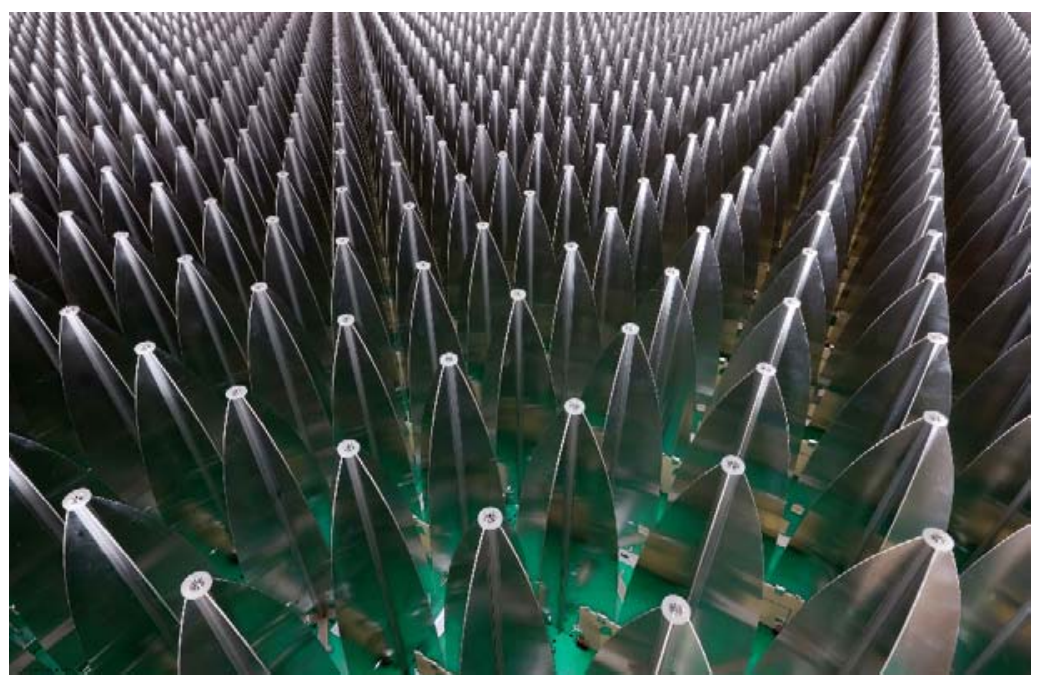

Figure 2 EMBRACE antenna field 


\subsection{Phased array feeds: ASKAP}

The Australian SKA Pathfinder (ASKAP) [8] is a new telescope that utilizes focal plane phased array feeds on $3612-\mathrm{m}$ antennas. The instrument will be deployed at a new radio-quiet observatory, the Murchison Radio-astronomy Observatory in the Midwest region of Western Australia. Table 4 gives a summary of the system specifications.

Table 4 Specifications ASKAP

\begin{tabular}{|l|l|}
\hline Number of dishes & Up to 45 \\
\hline Dish diameter & $12 \mathrm{~m}$ \\
\hline Max. baseline & $8 \mathrm{~km}$ \\
\hline Resolution & $8 ”$ \\
\hline Sensitivity & $110 \mathrm{~m}^{2} / \mathrm{K}$ \\
\hline Survey Speed & $5.5 \mathrm{e} 5 \mathrm{~m}^{4} / \mathrm{K}^{2} \mathrm{deg}^{2}$ \\
\hline Observing frequency & $700-1800 \mathrm{MHz}$ \\
\hline Field of View & $30 \mathrm{deg}^{2}$ \\
\hline Processed Bandwidth & $300 \mathrm{MHz}$ \\
\hline Channels & $16 \mathrm{k}$ \\
\hline Focal Plane Phased Array & 196 elements \\
\hline$T_{\text {sys }}$ & $35 \mathrm{~K}$ \\
\hline
\end{tabular}

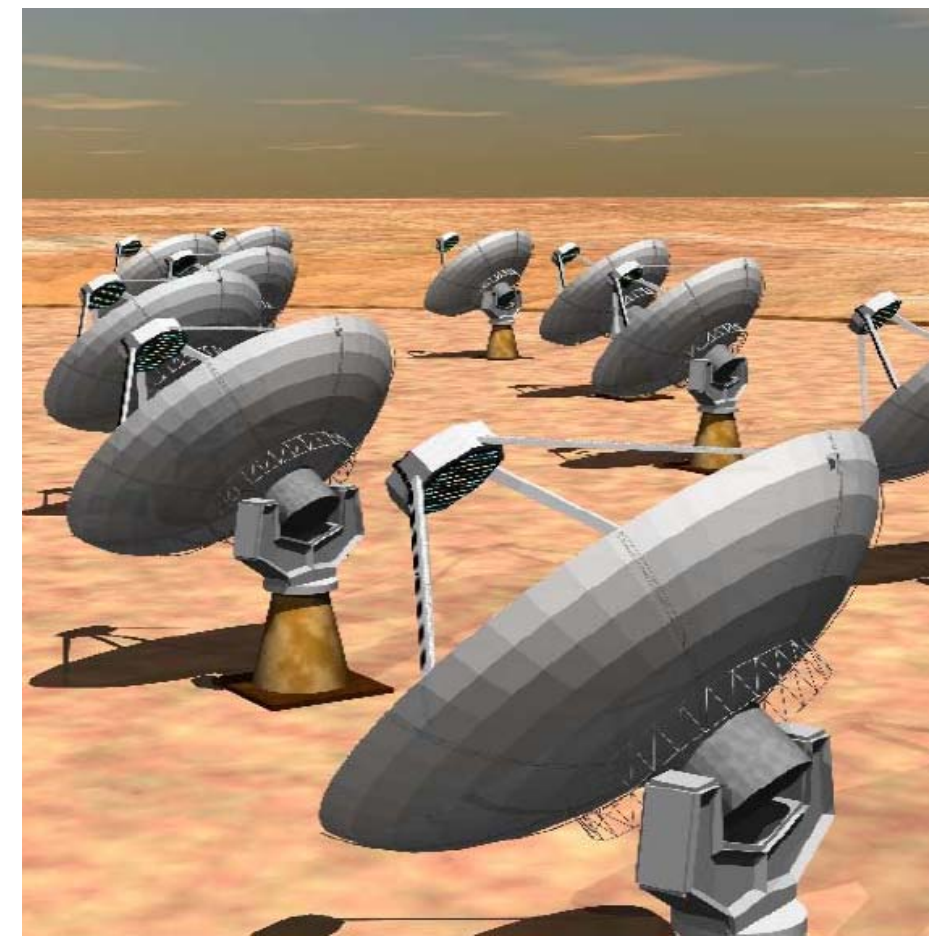

Figure 3 Artist impression ASKAP (CSIRO)

The Phased array feed system of a large array of antenna elements in the focus of the reflector. The signals of these elements are used to create so-called compound beams. These compound beams, a weighted sum of elements, can generate a very efficient reflector illumination and when building more beams, a large field of view can be achieved. A large patch of the sky can be observed in one instant. Figure 4 gives a simplified schematic where two beams are synthesized. 


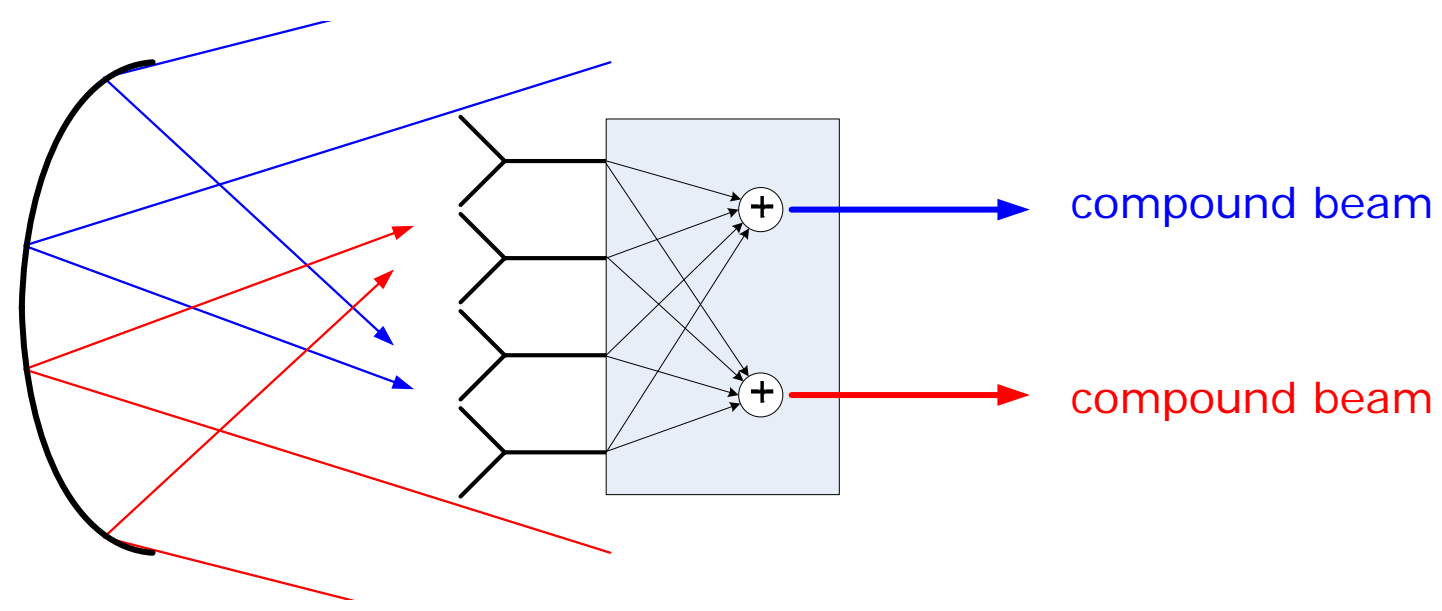

Figure 4 Phased array feeds

\subsection{Phased array feeds: APERTIF}

The Westerbork Synthesis Radio Telescope (WSRT) is currently equipped with a multi frequency front-end capable of observations in the frequency band $300 \mathrm{MHz}-8 \mathrm{GHz}$. These frequency bands are served with single horn or dipole antennas. In order to significantly improve the survey speed of the WSRT, a program has been started to replace the front-ends with a phased array feed system. Table 5 presents a summary of the system specification. With the Field of View improvement of the phased array system an improvement of survey speed of the order of 100 times will be achieved.

Table 5 APERTIF system specifications

\begin{tabular}{|l|l|}
\hline Number of dishes & 12 (total Westerbork array 14) \\
\hline Dish diameter & $25 \mathrm{~m}$ \\
\hline Sensitivity & $100 \mathrm{~m}^{2} / \mathrm{K}$ \\
\hline Observing frequency & $1000-1750 \mathrm{MHz}$ \\
\hline Field of View & $8 \mathrm{deg}^{2}$ \\
\hline Processed Bandwidth & $300 \mathrm{MHz}$ \\
\hline Focal Plane Phased Array & 112 elements \\
\hline $\mathrm{T}_{\text {sys }}$ & 50 kelvin \\
\hline
\end{tabular}

\section{Conclusion}

Significant R\&D effort will be devoted to the demonstrators and pathfinders that focus on WFoV technology for the next generation radio telescopes. Furthermore these systems are in it's self already interesting scientific instruments that will trigger a revolution in the capabilities of radio telescopes. 


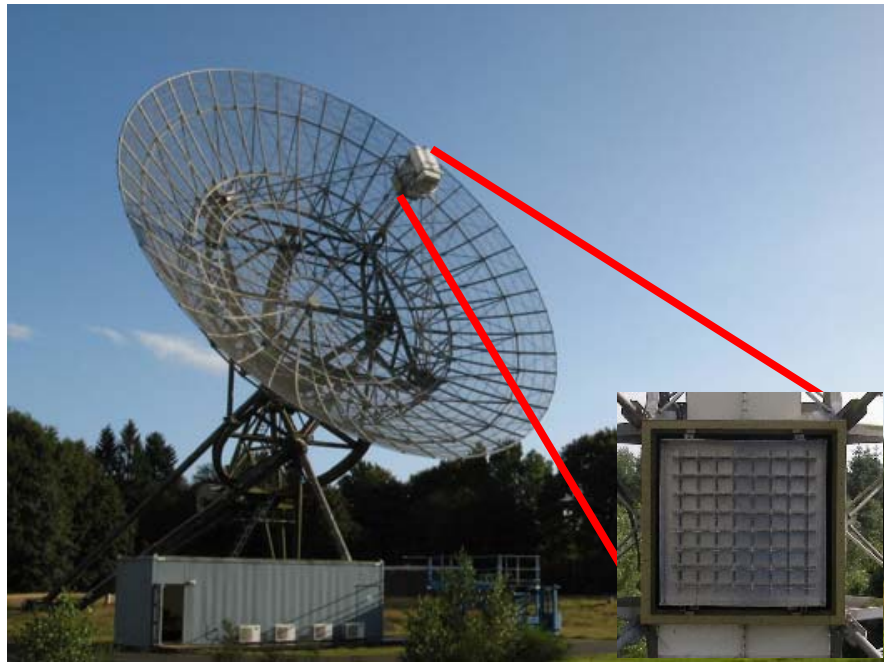

Figure 5 Westerbork dish equipped with APERTIF prototype

\section{Acknowledgement}

The following persons have contributed material to this paper: D. de Boer, CSIRO for ASKAP, A. Gunst, ASTRON for LOFAR, W. van Cappellen, ASTRON for APERTIF, D. Kant, ASTRON for EMBRACE, R. Schillizi, University of Manchester on SKA.

The SKADS work has been supported by EU-FP6 SKADS 011938.

\section{References}

[1] C. Carilli, S. Rawlings (eds.), Science with the Square Kilometre Array, New Astronomy Reviews 28, 2004, Elsevier, ISSN 1387-6473

[2] H.R. Klöckner, S. Rawlings, M. Jarvis, A. Taylor (eds.), Cosmology, Galaxy Formation and Astroparticle Physics on the Pathway to the SKA, ISBN 978-90-805434-4-7

[3] www.skatelescope.org

[4] P.E. Dewdney, P.J. Hall, R.T. Schilizzi, T.J. Lazio, The Square Kilometre Array, Proceedings of the IEEE, Vol. 97, No.8, August 2009

[5] M. de Vos, A.W. Gunst, R. Nijboer, The LOFAR Telescope: System Architecture and Signal Processing, Proceedings of the IEEE, Vol. 97, No.8, August 2009

[6] www.skads-eu.org

[7] A. van Ardenne et.al., Extending the Field of View with Phased Array Techniques: Results of European SKA Research, Proceedings of the IEEE, Vol. 97, No.8, August 2009

[8] D. DeBoer et. al., Australian SKA Pathfinder: A High-Dynamic Range Wide-Field of View Survey Telescope, Proceedings of the IEEE, Vol. 97, No.8, August 2009 